\title{
On paranorm intuitionistic fuzzy I-convergent sequence spaces defined by compact operator
}

\author{
Vakeel A. Khan ${ }^{1}$, Yasmeen Khan ${ }^{1}$, Henna Altaf ${ }^{1}$, Ayhan Esi ${ }^{2, *}$, Ayaz Ahamd ${ }^{3}$ \\ ${ }^{1}$ Department of Mathematics, Aligarh Muslim University, Aligarh-202002, India \\ ${ }^{2}$ Department of Mathematics, Adiyaman University, Adiyaman, Turkey \\ ${ }^{3}$ Department of Mathematics, National Institute of Technology, Patna, India
}

\section{A R T I C L E IN F O}

\section{Article history:}

Received 29 December 2016

Received in revised form

19 March 2017

Accepted 23 March 2017

Keywords:

I-convergence

Fuzzy set

Bounded linear operator

Compact linear operator

Paranorm

\section{Introduction}

After the pioneering work of Zadeh (1965), a huge number of research papers have appeared on fuzzy theory and its applications as well as fuzzy analogues of the classical theories. Fuzzy set theory is a powerful hand set for modelling uncertainty and vagueness in various problems arising in field of science and engineering. It has a wide range of applications in various fields: population dynamics (Barros et al., 2000), chaos control (Fradkov and Evans, 2005), computer programming (Giles, 1980), nonlinear dynamical system (Hong and Sun, 2006), etc. Fuzzy topology is one of the most important and useful tools and it proves to be very useful for dealing with such situations where the use of classical theories breaks down. The concept of intuitionistic fuzzy normed space (Saadati and Park, 2006) and of intuitionistic fuzzy 2-normed space (Mursaleen and Lohani, 2009) is the latest developments in fuzzy topology. Khan et al. (2014, 2015, 2017) and Khan and Yasmeen (2016a,b,c) studied the intuitionistic fuzzy zweier I-convergent sequence spaces defined by paranorm, modulus function and Orlicz function.

The notion of statistical convergence is a very useful functional tool for studying the convergence problems of numerical problems/matrices (double

\footnotetext{
* Corresponding Author.

Email Address: aesi23@hotmail.com (A. Esi)

https://doi.org/10.21833/ijaas.2017.05.024

2313-626X/C) 2017 The Authors. Published by IASE.

This is an open access article under the CC BY-NC-ND license

(http://creativecommons.org/licenses/by-nc-nd/4.0/)
}

sequences) through the concept of density. The notion of I-convergence, which is a generalization of statistical convergence (Fast, 1951; Esi and Özdemir, 2016; Mursaleen and Mohiuddine, 2009a;b;2010; Hazarika and Mohiuddine, 2013; Alotaibi et al., 2014; Mohiuddine and Lohani, 2009; Mursaleen et al., 2010) was introduced by Kostyrko et al. (2000) by using the idea of I of subsets of the set of natural numbers $\mathbb{N}$ and further studied in (Nabiev et al., 2007). Recently, the notion of statistical convergence of double sequences $\mathrm{x}=\left(x_{i j}\right)$ has been defined and studied by Edely (2003), and for fuzzy numbers by Savas (2004), Mursaleen et al. (2016). Quite recently, Das et al. (2008) studied the notion of I and Iconvergence of double sequences in $\mathbb{R}$.

We recall some notations and basic definitions used in this paper.

Definition 1.1: A binary operation $*:[0,1] \times[0,1] \rightarrow$ $[0,1]$ is said to be continuous t-norm, if the following hold:

1.* is associative and commutative,

2 . $*$ is continuous,

3. $x * 1=x$ for all $x \in[0,1]$,

4. $x * y \leq z * w$ whenever $x \leq z$ and $y \leq w$ where $x, y, z, w \in[0,1]$.

Example 1.1: Define $x * y=x . y$ where the usual multiplication is. Then it can be shown that $*$ is a continuous t-norm.

Definition 1.2: A binary operation $\diamond:[0,1] \times[0,1] \rightarrow$ $[0,1]$ is said to be continuous t-norm, if it satisfies the following properties: 
1. $\diamond$ is associative and commutative,

2. $\diamond$ is continuous,

3. $x \diamond 0=x$ for all $x \in[0,1]$,

4. $x \diamond y \leq z \diamond w$ whenever $x \leq z$ and $y \leq w$ where $x, y, z, w \in[0,1]$.

Example 1.2: $x \diamond y=\min \{x+y, 1\}$ is a continuous t-norm.

Definition 1.3: Let $X$ be a non-empty set. A subsets $I$ of $X$ is said to be an ideal if,

1. $A, B \in I \Rightarrow A \cup B \in I$;

2. $A \in I, B \subseteq A \Rightarrow B \in I$.

The above properties are called additivity and hereditary respectively. An Ideal $I$ is called nontrivial if $X \neq I$.

Definition.1.4: Let $X$ be a non-empty set. Then $\mathcal{F} \subseteq$ $2 x$ is said to be filter on $X$ if,

1. $\emptyset \notin \mathcal{F}$

2. $A, B \in \mathcal{F} \Rightarrow A \cap B \in \mathcal{F}$,

3. $A \in \mathcal{F}$ and $A \subseteq B \Rightarrow B \in \mathcal{F}$.

To each ideal $I$, a filter $\mathcal{F}$ is associated defined as $\mathcal{F}(I)=\{M \subseteq X: M c \in I\}$.

Definition 1.5: Let $I \subseteq 2 \mathbb{N}$ be a non-trivial ideal in $\mathbb{N}$. Then a sequence $x=\left(x_{k}\right)$ is said to be $I$ convergent to a number $L \in \mathbb{R}$ if, for every $\epsilon>0$, the set

$\left\{k \in \mathbb{N}:\left|x_{k}-L\right|\right\} \in I$.

Definition 1.6: Let $I \subseteq 2 \mathbb{N}$ be a non-trivial ideal in $\mathbb{N}$. Then a sequence $x=\left(x_{k}\right)$ is said to be $I$ Cauchy if, for each $\epsilon>0$, there exists a number $N=$ $N(\epsilon)$ such that the set $\left\{k \in \mathbb{N}:\left|x_{K}-x_{N}\right| \geq \epsilon\right\} \in I$.

Definition 1.7: Let $X$ be a non-empty set. A fuzzy set $A$ in $X$ is characterized by its membership function:

$\mu_{A}: A \rightarrow[0,1]$

and $\mu_{A}(x)$ is called as the degree of membership of element $x$ in fuzzy set $A$ for each $x$ in $X$.

Definition 1.8: The five-tuple $(X, \mu, v, *, \diamond)$ is said to be an intuitionistic fuzzy normed space(IFNS) if $X$ is a vector space, $*$ is a continuous t-norm, $\diamond$ is a continuous t-conorm and $\mu, v$ are fuzzy sets on $X \times$ $(0, \infty)$ satisfying the following conditions for every $x, y \in X$ and $s, t>0$ (Khan et al., 2015):

a) $\mu(x, t)+v(x, t) \leq 1$,

b) $\mu(x, t)>0$,

c) $\mu(x, t)=1$ if and only if $x=0$

d) $\mu(\alpha x, t)=\mu\left(x, \frac{t}{|\alpha|}\right)$ for each $\alpha \neq 0$

e) $\mu(x, t) * \mu(y, s) \leq \mu(x+y, t+s)$

f) $\mu(x, t) *(0, \infty) \rightarrow[0,1]$ is continuous,

g) $\lim _{t \rightarrow \infty} \mu(x, t)=1$ and $\lim _{t \rightarrow 0} \mu(x, t)=0$,

h) $v(x, t) \leq 1$, i) $v(x, t)=0$ if and only if $x=0$,

j) $v(\alpha x, t)=v\left(x, \frac{t}{|\alpha|}\right)$ for each $\alpha \neq 0$,

k) $v(x, t) \diamond v(y, s) \geq v(x+y, t+s)$,

l) $v(x,):.(0, \infty) \rightarrow[0,1]$ is continuous

m) $\lim _{t \rightarrow \infty} v(x, t)=0$ and $\lim _{t \rightarrow 0} v(x, t)=1$.

In this case $(\mu, v)$ is called an intuitionistic fuzzy norm.

Definition 1.9: Let $(X, \mu, v, *, \diamond)$ be IFNS then the sequence $x=\left(x_{k}\right)$ is said to be convergent to continuous $L \in X$ with respect to the intuitionistic fuzzy norm $(\mu, v)$ if, for every $\epsilon>0$ and $t>0$, there exists $k_{0} \in \mathbb{N}$ such that $\mu\left(x_{k}-L, t\right)>1-\epsilon$ and $v$ $\left(x_{k}-L, t\right)<\epsilon$ for all $k \geq k_{0}$. In this case we write $(\mu, v)-\lim x=L$.

Definition 1.10: Let $(X, \mu, v, *, \diamond)$ be IFNS then the sequence $x=\left(x_{k}\right)$ is said to be Cauchy sequence with respect to the intuitionistic fuzzy norm $(\mu, v)$ if, for every $\epsilon>0$ and $t>0$, there exists $k_{0} \in \mathbb{N}$ such that $\mu\left(x_{k}-x_{l}, t\right)>1-\epsilon$ and $v\left(x_{k}-x_{l}, t\right)<\epsilon$ for all $k, l \geq k_{0}$.

Definition 1.11: Let $K$ be the subset of natural numbers $\mathbb{N}$. Then the asymptotic density of $K$, denoted by $\delta(K)$, is defined as $\delta(K)=\lim _{n} \frac{1}{n} \mid\{k \leq$ $n: k \in K\} \mid$, where the vertical bars denote the cardinality of the enclosed set.

Definition 1.12: A number sequence $x=\left(x_{k}\right)$ is said to be statistically convergent to a number $\ell$ if, for each $\epsilon>0$, the set $K(\epsilon)=\left\{k \leq n:\left|x_{k}-\ell\right|\right\} \geq \epsilon$ has asymptotic density zero, i.e. $\lim _{n} \frac{1}{n} \mid\{k \leq$ $\left.\left.n:\left|x_{k}-\ell\right|\right\} \geq \epsilon\right\} \mid=0$, In this case we write st$\lim x=\ell$.

Definition 1.13: A number sequence $x=\left(x_{k}\right)$ is said to be statistically Cauchy convergent if, for every $\epsilon>0$, there exists a number $N=N(\epsilon)$ such that

$\left.\lim _{n} \frac{1}{n} \mid\left\{j \leq n:\left|x_{j}-x_{N}\right|\right\} \geq \epsilon\right\} \mid=0$.

The concepts of statistical convergence and statistical Cauchy for double sequences in intuitionistic fuzzy normed spaces have been studied by Mursaleen et al. (2010).

Definition 1.14: Let $I \subseteq 2 \mathbb{N}$ be a non-trivial ideal and $(X, \mu, v, *, \diamond)$ be an IFNS then the sequence $x=$ $\left(x_{k}\right)$ of elements of $X$ is said to be $I$-convergent to $L \in$ $X$ with respect to the intuitionistic fuzzy norm $(\mu, v)$ if for every $\epsilon>0$ and $t>0$, the set

$\left\{k \in \mathbb{N}: \mu\left(x_{k}-L, t\right) \geq 1-\epsilon\right.$ or $\left.v\left(x_{k}-L, t\right) \leq \epsilon\right\} \in I$.

In this case $L$ is called the $I$-limit of the sequence $\left(x_{k}\right)$ with respect to the intuitionistic fuzzy norm $(\mu, v)$ and $I_{(\mu, v)}-\lim \left(x_{k}\right)=L$.

Definition 1.15: Let $X$ and $Y$ be two normed linear spaces and $T: D(T) \rightarrow Y$ be a linear operator, where 
$D \subset X$ Then the operator $T$ is said to be bounded, if there exists a positive real $k$ such that (Khan et al., 2015)

$\|T x\| \leq k\|x\|$, for all $x \in D(T)$.

The set of all bounded linear operators $B(X ; Y)$ (Kreyszig, 1989) is a normed linear space normed by

$\|T\|=\sup _{x \in X,\|x\|=1}\|T x\|$

and $B(X, Y)$ is a Banach space if $Y$ is a Banach space.

Definition 1.16: Let $X$ and $Y$ be two normed linear spaces. An operator $T: X \rightarrow Y$ is said to be a compact linear operator (or completely continuous linear operator) if (Khan et al., 2015),

1. $T$ is linear,

2. $T$ maps every bounded sequence $\left(x_{k}\right)$ in $X$ on to a sequence $\left(T\left(x_{k}\right)\right)$ in $Y$ which has a convergent subsequence.

The set of all compact linear operators $C(X, Y)$ is a closed subspace of $B(X, Y)$ and $C(X, Y)$ is Banach space, if $Y$ is a Banach space.

Definition 1.17: Let $X$ is a vector space. A function $p: X \rightarrow \mathbb{R}$ is said to be a paranorm if $p$ satisfies the following conditions:

1. $p(x) \geq 0$, for all $x \in X$,

2. $p(-x)=p(x)$, for all $x \in X$,

3. $p(x+y) \leq p(x)+p(y)$, for all $x, y \in X$,

4. if $\left(\lambda_{n}\right)$ is a sequence of scalars with $\left(\lambda_{n}\right) \rightarrow(\lambda)$ as $(n \rightarrow$ $\infty)$ and $\left(x_{n}\right)$ is a sequence

of vectors such that $p\left(x_{n}-x\right) \rightarrow 0$ as $(n \rightarrow$ $\infty)$ then $p\left(\lambda_{n} x_{n}-\lambda x\right) \rightarrow 0$ as $n \rightarrow \infty$.

In this article we introduce the following sequence spaces:

$S_{(\mu, v)}^{I}(T)(p)=\left\{\left(x_{k}\right) \in \ell_{\infty}:\left\{k \in \mathbb{N}: \mu\left[T\left(x_{k}-L, t\right)\right]^{p_{k}} \leq 1-\right.\right.$ $\left.\left.\epsilon \operatorname{Or} v\left[T\left(x_{k}\right)-L, t\right]^{p_{k}} \geq \epsilon\right\} \in I\right\}$,

$S_{0(\mu, v)}^{I}(T)(p)=\left\{\left(x_{k}\right) \in \ell_{\infty}:\left\{k \in \mathbb{N}: \mu\left[T\left(x_{k}\right), t\right]^{p_{k}} \leq 1-\right.\right.$ $\epsilon$ Or $\left.\left.v\left[T\left(x_{k}\right), t\right]^{p_{k}} \geq \epsilon\right\} \in I\right\}$.

We also define an open ball with center $x$ and radius $r$ with respect to $t$ as follows:

$B_{X}(r, t)(T)(p)=\left\{\left(y_{k}\right) \in\right.$

$\left.\ell_{\infty}:\left\{\begin{array}{c}k \in \mathbb{N}: \mu\left[T\left(x_{k}\right)-T\left(y_{k}\right), t\right]^{p_{k}} \leq 1-\epsilon \\ 0 \mathrm{r} v\left[T\left(x_{k}\right)-T\left(y_{k}\right), t\right]^{p_{k}} \geq \epsilon\end{array}\right\} \in I\right\}$.

\section{Main results}

Theorem 2.1: $S_{(\mu, v)}^{I}(T)(p)$ and $S_{0(\mu, v)}^{I}(T)(p)$ are vector spaces.

Proof: We shall prove the result for $S_{(\mu, v)}^{I}(T)(p)$. The proof for the other space will follow similarly.
Let $x=\left(x_{k}\right), y=\left(y_{k}\right) \in S_{(\mu, v)}^{I}(T)(p)$ and $\alpha, \beta$ be scalars. Then for given $\epsilon>0$, we have

$A_{1}=\left\{k \in \mathbb{N}: \mu\left[T\left(x_{k}\right)-L_{1}, \frac{t}{2|\alpha|}\right]^{p_{k}} \leq 1-\epsilon\right.$ or $v\left[T\left(x_{k}\right)-\right.$ $\left.\left.L_{1}, \frac{t}{2|\alpha|}\right]^{p_{k}} \geq \epsilon\right\} \in I$,

$A_{2}=\left\{k \in \mathbb{N}: \mu\left[T\left(y_{k}\right)-L_{2}, \frac{t}{2|\beta|}\right]^{p_{k}} \leq 1-\epsilon\right.$ or $v\left[T\left(y_{k}\right)-\right.$ $\left.\left.L_{2}, \frac{t}{2|\beta|}\right]^{p_{k}} \geq \epsilon\right\} \in I$.

$A_{1}^{c}=\left\{k \in \mathbb{N}: \mu\left[T\left(x_{k}\right)-L_{1}, \frac{t}{2|\alpha|}\right]^{p_{k}}>1-\epsilon\right.$ or $v\left[T\left(x_{k}\right)-\right.$ $\left.\left.L_{1}, \frac{t}{2|\alpha|}\right]^{p_{k}}<\epsilon\right\} \in \mathcal{F}(I)$

$A_{2}^{c}=\left\{k \in \mathbb{N}: \mu\left[T\left(y_{k}\right)-L_{2}, \frac{t}{2|\beta|}\right]^{p_{k}}>1-\right.$

$\epsilon$ or $\left.v\left[T\left(y_{k}\right)-L_{2}, \frac{t}{2|\beta|}\right]^{p_{k}}<\epsilon\right\} \in \mathcal{F}(I)$.

Define the set $A 3=A 1 \cup A 2$, so that $A 3 \in I$. It follows that $A_{3}^{c}$ is a non-empty set in $\mathcal{F}(I)$. We shall show that for each $\left(x_{k}\right),\left(y_{k}\right) \in S_{(\mu, v)}^{I}(T)(p)$,

$A_{3}^{c} \subset\left\{\begin{array}{c}\mu\left[\alpha T\left(x_{k}\right)+\beta T\left(y_{k}\right)-\left(\alpha L_{1}+\beta L_{2}\right), t\right]^{p_{k}}>1-\epsilon \\ \text { or } \\ v\left[\alpha T\left(x_{k}\right)+\beta T\left(y_{k}\right)-\left(\alpha L_{1}+\beta L_{2}\right), t\right]^{p_{k}}<\epsilon\end{array}\right\}$.

Let $m \in A_{3}^{c}$, then we have,

$\mu\left[T\left(x_{m}\right)-L_{1}, \frac{t}{2|\alpha|}\right]^{p_{k}}>1-\epsilon$ or $v\left[T\left(x_{m}\right)-L_{1}, \frac{t}{2|\alpha|}\right]^{p_{k}}<$ $\epsilon$

and

$\quad \mu\left[T\left(y_{m}\right)-L_{2}, \frac{t}{2|\beta|}\right]^{p_{k}}>1-\epsilon$ or $v\left[T\left(y_{m}\right)-\right.$
$\left.L_{2}, \frac{t}{2|\beta|}\right]^{p_{k}}<\epsilon$.

We have

$\mu\left[\left(\alpha T\left(x_{m}\right)-\beta T\left(y_{m}\right)\right)-\left(\alpha L_{1}+\beta L_{2}\right)\right]^{p_{k}} \geq \mu\left[\alpha T\left(x_{m}\right)-\right.$ $\left.\alpha L_{1}, \frac{t}{2}\right]^{p_{k}} * \mu\left[\beta T\left(x_{m}\right)-\beta L_{2}, \frac{t}{2}\right]^{p_{k}}$

$=\mu\left[T\left(x_{m}\right)-L_{1}, \frac{t}{2|\alpha|}\right]^{p_{k}} * \mu\left[T\left(x_{m}\right)-L_{2}, \frac{t}{2|\beta|}\right]^{p_{k}}$

$>(1-\epsilon) *(1-\epsilon)=1-\epsilon$.

and

$v\left[\alpha T\left(x_{m}\right)+\beta T\left(y_{m}\right)-\left(\alpha L_{1}+\beta L_{2}\right), t\right]^{p_{k}} \leq v\left[\alpha T\left(x_{m}\right)-\right.$ $\left.\alpha L_{1}, \frac{t}{2}\right]^{p_{k}} \diamond v\left[\beta T\left(x_{m}\right)-\beta L_{2}, \frac{t}{2}\right]^{p_{k}}$

$=v\left[T\left(x_{m}\right)-\alpha L_{1}, \frac{t}{2|\alpha|}\right]^{p_{k}} \diamond v\left[T\left(x_{m}\right)-L_{2}, \frac{t}{2|\beta|}\right]^{p_{k}}$

$<\epsilon \diamond \epsilon=\epsilon$.

Therefore

$A_{3}^{c} \subset$

$\left\{\begin{array}{c}k \in \mathbb{N}: \mu\left[\alpha T\left(x_{k}\right)+\beta T\left(y_{k}\right)-\left(\alpha L_{1}+\beta L_{2}\right), t\right]^{p_{k}}>1-\epsilon \\ \text { or } v\left[\alpha T\left(x_{k}\right)+\beta T\left(y_{k}\right)-\left(\alpha L_{1}+\beta L_{2}\right), t\right]^{p_{k}}<\epsilon\end{array}\right\}=$ $B$ (say).

So we have $B^{c} \subset A_{3} \in I$ which proves $S_{(\mu, v)}^{I}(T)(p)$ is a linear space. 
Theorem 2.2: Every open ball $B_{x}(r, t)(T)(p)$ is an open set in $S_{(\mu, v)}^{I}(T)(p)$.

Proof: Let $B_{x}(r, t)(T)(\mathcal{P})$ be an open ball with center $x$ and radius $r$ with respect to $t$. That is

$$
\begin{aligned}
& B_{x}(r, t)(T)(p)= \\
& \left\{\begin{array}{c}
y=\left(y_{k}\right) \in \ell_{\infty}:\left\{k \in \mathbb{N}: \mu\left[T\left(x_{k}\right)-T\left(y_{k}\right), t\right]^{p_{k}} \leq 1-r\right. \\
\text { or } \left.v\left[T\left(x_{k}\right)-T\left(y_{k}\right), t\right]^{p_{k}} \geq r\right\} \in I
\end{array}\right\}
\end{aligned}
$$

\section{Let $y \in B_{x}^{c}(r, t)(T)(p)$. Then}

$\mu\left[T\left(x_{k}\right)-T(y), t\right]^{p_{k}}>1-r$ and $\quad v\left[T\left(x_{k}\right)-T\left(y_{k}\right), t\right]^{p_{k}}<$ $r$.

Since $\mu\left[T\left(x_{k}\right)-T\left(y_{k}\right), t\right]^{p_{k}}>1-r$ there exists $t_{0} \in$ $(0,1)$ such that $\mu\left[T\left(x_{k}\right)-T\left(y_{k}\right), t_{0}\right]^{p_{k}}>1-r$ and $v\left[T\left(x_{k}\right)-T\left(y_{k}\right), t_{0}\right]^{p_{k}}<r . \quad \quad$ Putting $r_{o}=$ $\mu\left[T\left(x_{k}\right)-T\left(y_{k}\right), t_{0}\right]^{p_{k}}$, we have $r_{0}>1-r$ there exists $s \in(0,1)$ such that $s<r$ and hence $r_{0}>1-$ $s>1-r$. For $r_{0}>1-s$, we have $r_{1}, r_{2} \in(0,1)$ with $r_{1}, r_{2}>r_{0}$ and thus $r_{0} * r_{1}>1-s$ and $\left(1-r_{0}\right) \diamond$ $\left(1-r_{0}\right) \leq s$. Let $r_{3}=\max \left\{r_{1}, r_{2}\right\}$ and consider the ball $B_{y}^{c}\left(1-r_{3}, t-t_{0}\right)(T)(p)$. We proof that

$$
B_{y}^{c}\left(1-r_{3}, t-t_{0}\right)(T)(p) \subset B_{x}^{c}(r, t)(T)(p) .
$$

Let $z=\left(z_{k}\right) \in B_{y}^{c}\left(1-r_{3}, t-t_{0}\right)(T)(p)$. Then $\mu\left[T\left(y_{k}\right)-T\left(z_{k}\right), t-t_{0}\right]^{p_{k}}>r_{3}$ and $v\left[T\left(y_{k}\right)-T\left(z_{k}\right), t-\right.$ $\left.t_{0}\right]^{p_{k}}<1-r_{3}$.

Thus

$\mu\left[T\left(x_{k}\right)-T\left(z_{k}\right), t\right]^{p_{k}}>\mu\left[T\left(x_{k}\right)-T\left(y_{k}\right), t_{0}\right]^{p_{k} *}$ $\mu\left[T\left(y_{k}\right)-T\left(z_{k}\right), t-t_{0}\right]^{p_{k}}$

$\geq\left(r_{0} * r_{3}\right) \geq\left(r_{0} * r_{1}\right) \geq(1-s)>(1-r)$

and

$v\left[T\left(x_{k}\right)-T\left(z_{k}\right), t\right]^{p k} \leq v\left[T\left(x_{k}\right)-T\left(y_{k}\right), t_{0}\right]^{p_{k}} 。$ $v\left[T\left(y_{k}\right)-T\left(z_{k}\right), t-t_{0}\right]^{p_{k}}$ $\leq\left(1-r_{0}\right) \bullet\left(1-r_{3}\right) \leq\left(1-r_{0}\right) \diamond\left(1-r_{2}\right) \leq s<r$.

Thus $z \in B_{x}^{c}(r, t)(T)(p)$ and hence

$B_{y}^{c}\left(1-r_{3}, t-t_{0}\right)(T)(p) \subset B_{x}^{c}(r, t)(T)(p)$.

Remark 2.1: $S_{(\mu, v)}^{I}(T)(p)$ is an IFNS.

Define $\tau_{(\mu, v)}^{I}(T)(p)=\left\{A \subset S_{(\mu, v)}^{I}(T)(p)\right.$ : for each $x \in A$ there exists $t>0$ and $r \in(0,1)$. Such that $\left.B_{x}(r, t)(T)(p) \subset A\right\}$. Then $\tau_{(\mu, v)}^{I}(T)(p)$ is a topology on $S_{(\mu, v)}^{I}(T)(p)$.

Theorem 2.3: The topology $\tau_{(\mu, v)}^{I}(T)(p)$ on $S_{0(\mu, v)}^{I}(T)(p)$ is first countable.

Proof: $\left\{B_{x}\left(\frac{1}{n}, \frac{1}{n}\right)(T)(p): n=1,2,3, \ldots \ldots\right\}$ is a local base at $x$. Hence the topology $\tau_{(\mu, v)}^{I}(T)(p)$ on $S_{0(\mu, v)}^{I}(T)(p)$ is first countable.
Theorem 2.4: $S_{(\mu, v)}^{I}(T)(p)$ and $S_{0(\mu, v)}^{I}(T)(p)$ are Hausdorrf spaces.

Proof: We prove the result for $S_{(\mu, v)}^{I}(T)(p)$. The proof for $S_{0(\mu, v)}^{I}(T)(p)$ will follow on similar lines. Let $x, y \in S_{(\mu, v)}^{I}(T)(p)$ such that $x \neq y$. Then $0<$ $\mu[T(x)-T(y)]^{p_{k}}<1$ and $0<v[T(x)-T(y)]^{p_{k}}<1$. Put $r_{1}=\mu[T(x)-T(y)]^{p_{k}}, r_{2}=v[T(x)-T(y), t]^{p_{k}}$ and $r=\max \left\{r_{1}, 1-r_{2}\right\}$. For each $r_{0} \in(r, 1)$ There exists $r_{3}$ and $r_{4}$ with $r_{3}, r_{4}>r$ and hence $r_{3} * r_{4} \geq r_{0}$ and $\left(1-r_{3}\right) \diamond\left(1-r_{4}\right) \leq\left(1-r_{0}\right)$. Let $r_{5}=$ $\max \left\{r_{3}, 1-r_{4}\right\}$, then we can show the open balls $B_{x}^{c}\left(1-r_{5}, \frac{t}{2}\right)$ and $B_{y}^{c}\left(1-r_{5}, \frac{t}{2}\right)$ are disjoint. Suppose on contrary there exists

$z \in B_{x}^{c}\left(1-r_{5}, \frac{t}{2}\right) \cap B_{y}^{c}\left(1-r_{5}, \frac{t}{2}\right)$,

then

$r_{1}=\mu[T(x)-T(y), t]^{p_{k}} \geq \mu\left[T(x)-T(z), \frac{t}{2}\right]^{p_{k}} *$

$\mu\left[T(z)-T(y), \frac{t}{2}\right]^{p_{k}}$

$>r_{5} * r_{5} \geq r_{3} * r_{3} \geq r_{0}>r_{1}$

and

$r_{2}=v[T(x)-T(y), t]^{p_{k}} \leq v\left[T(x)-T(z), \frac{t}{2}\right]^{p_{k}} 。$

$v\left[T(z)-T(y), \frac{t}{2}\right]^{p_{k}}$

$<\left(1-r_{5}\right) \diamond\left(1-r_{5}\right) \leq\left(1-r_{3}\right) \diamond\left(1-r_{3}\right)$

$\leq\left(1-r_{0}\right)<r_{2}$

which is contradiction. Hence $S_{(\mu, v)}^{I}(T)(p)$ is Hausdorrf spaces.

Theorem 2.5: $S_{(\mu, v)}^{I}(T)(p)$ is IFNS and $\tau_{(\mu, v)}^{I}(T)(p)$ is topology on $S_{(\mu, v)}^{I}(T)(p)$. Then a sequence $x=\left(x_{k}\right) \in S_{(\mu, v)}^{I}(T)(p), x_{k} \rightarrow x$ if and only if $\mu\left[T\left(x_{k}\right)-T(x), t\right]^{p_{k}} \rightarrow 1$ and

$v\left[T\left(x_{k}\right)-T(x), t\right]^{p_{k}} \rightarrow 0$ as $k \rightarrow \infty$.

Proof: Fix $t_{0}>0$. suppose $x_{k} \rightarrow x$ as $(k \rightarrow \infty)$. Then for $r \in(0,1)$, there exists $n_{0} \in \mathbb{N}$ such that $\left(x_{k}\right) \in B_{x}(r, t)(T)(p)$ for all $k \geq n_{0}$.

$B_{x}(r, t)(T)(p)=\left\{k \in \mathbb{N}: \mu\left[T\left(x_{k}\right)-T(x), t\right]^{p_{k}} \leq 1-\right.$ $r$ or $\left.v\left[T\left(x_{k}\right)-T(x), t\right]^{p_{k}} \geq r\right\} \in I$,

and so $B_{x}^{c}(r, t)(T)(p) \in F(I)$. Then $1-\mu\left[T\left(x_{k}\right)-\right.$ $T(x), t]^{p_{k}}<r$ and $v\left[T\left(x_{k}\right)-T(x), t\right]^{p_{k}}<r$. Hence $\mu\left[T\left(x_{k}\right)-T(x), t\right]^{p_{k}} \rightarrow 1$ and $v\left[T\left(x_{k}\right)-\right.$ $T(x), t]^{p_{k}} \rightarrow 0$ as $k \rightarrow \infty$.

Conversely, if for each $t>0 \quad \mu\left[T\left(x_{k}\right)-\right.$ $T(x), t]^{p_{k}} \rightarrow 1$ and $v\left[T\left(x_{k}\right)-T(x), t\right]^{p_{k}} \rightarrow 0$ as $k \rightarrow$ $\infty$, then $r \in(1,0)$, there exists $n_{0} \in \mathbb{N}$ such that $1-$ $\mu\left[T\left(x_{k}\right)-T(x), t\right]^{p_{k}}<r$ and $v\left[T\left(x_{k}\right)-T(x), t\right]^{p k}<$ $r$ for all $k \geq n_{0}$. It follows that $\mu\left[T\left(x_{k}\right)-\right.$ $T(x), t]^{p k}>1-r$ and $v\left[T\left(x_{k}\right)-T(x), t\right]^{p k}<r$ for all $k \geq n_{0}$. Thus $\left(x_{k}\right) \in B_{x}^{c}(r, t)(T)(p)$ for all $k \geq n_{0}$ and hence $x_{k} \rightarrow x$. 
Theorem 2.6: A Sequence $x=\left(x_{k}\right) \in S_{(\mu, v)}^{I}(T)(p)$ is $I$-convergent if and only if for every $\epsilon>0$ and $t>0$ there exists a number $N=N(x, \epsilon, t)$ such that

$\left\{k \in \mathbb{N}: \mu\left[T\left(x_{k}\right)-L, \frac{t}{2}\right]^{p_{k}}>1-\epsilon\right.$ or $v\left[T\left(x_{k}\right)-L, \frac{t}{2}\right]^{p_{k}}<$ $\epsilon\} \in \mathcal{F}(I)$

Proof: Suppose that $I_{(\mu, v)}-\lim x=L$ and let $\epsilon>0$ and $t>0$. For a given $\epsilon>0$, choose $s>0$ such that $(1-\epsilon) *(1-\epsilon)>1-s$ and $\epsilon \diamond \epsilon<s$. Then for each $x \in S_{(\mu, v)}^{I}(T)(p)$

$A=\left\{k \in \mathbb{N}: \mu\left[T\left(x_{k}\right)-L, \frac{t}{2}\right]^{p_{k}} \leq 1-\epsilon\right.$ or $v\left[T\left(x_{k}\right)-\right.$ $\left.\left.L, \frac{t}{2}\right]^{p_{k}} \geq \epsilon\right\} \in I$

and thus

$A^{c}=\left\{k \in \mathbb{N}: \mu\left[T\left(x_{k}\right)-L, \frac{t}{2}\right]^{p_{k}}>1-\epsilon\right.$ or $v\left[T\left(x_{k}\right)-\right.$ $\left.\left.L, \frac{t}{2}\right]^{p_{k}}<\epsilon\right\} \in \mathcal{F}(I)$.

Conversely let us choose $N \in A$. Then

$\mu\left[T\left(x_{N}\right)-L, \frac{t}{2}\right]^{p_{k}}>1-\epsilon$ or $v\left[T\left(x_{N}\right)-L, \frac{t}{2}\right]^{p_{k}}<\epsilon$.

Now we want to show that there exists number $N=$ $N(x, \epsilon, t)$ such that

$\left\{k \in \mathbb{N}: \mu\left[T\left(x_{k}\right)-T\left(x_{N}\right), t\right]^{p_{k}} \leq 1-s\right.$ or $v\left[T\left(x_{k}\right)-\right.$ $\left.\left.T\left(x_{N}\right), t\right]^{p_{k}} \geq s\right\} \in I$.

For this, define for each $x \in S_{(\mu, v)}^{I}(T)(p)$

$\mathrm{B}=\left\{k \in \mathbb{N}: \mu\left[T\left(x_{k}\right)-T\left(x_{N}\right), t\right]^{p_{k}} \leq 1-s\right.$ or $v\left[T\left(x_{k}\right)-\right.$ $\left.\left.T\left(x_{N}\right), t\right]^{p_{k}} \geq s\right\}$.

Now we have to show that $B \subset A$. Suppose $B \subsetneq A$. Then there exists $n \in B$ and $n \notin A$. Therefore we have,

$\mu\left[T\left(x_{n}\right)-T\left(x_{N}\right), t\right]^{p_{k}} \leq 1-s$ and $\mu\left[T\left(x_{n}\right)-L, \frac{t}{2}\right]^{p_{k}}>$ $1-\epsilon$.

In particular $\mu\left[T\left(x_{N}\right)-L, \frac{t}{2}\right]^{p_{k}}>1-\epsilon$. Thus,

$1-s \geq \mu\left[T\left(x_{n}\right)-T\left(x_{N}\right), t\right]^{p_{k}} \geq \mu\left[T\left(x_{n}\right)-L, \frac{t}{2}\right]^{p_{k}} *$

$\mu\left[T\left(x_{n}\right)-L, \frac{t}{2}\right]^{p_{k}}$

$>(1-\epsilon) *(1-\epsilon)>1-s$.

Which is not possible. Also we have,

$v\left[T\left(x_{n}\right)-T\left(x_{N}\right), t\right]^{p_{k}} \geq s$ and $v\left[T\left(x_{n}\right)-L, \frac{t}{2}\right]^{p_{k}}<\epsilon$.

In particular $v\left[T\left(x_{N}\right)-L, \frac{t}{2}\right]^{p_{k}}<\epsilon$. Thus,

$s \leq \mu\left[T\left(x_{n}\right)-T\left(x_{N}\right), t\right]^{p_{k}} \leq v\left[T\left(x_{n}\right)-L, \frac{t}{2}\right]^{p_{k}} 。$

$v\left[T\left(x_{N}\right)-L, \frac{t}{2}\right]^{p_{k}}$

$<\epsilon \diamond \epsilon<s$, which is not possible. Hence $B \subset A$. $A \in I$ implies $B \in$ I.

\section{Conclusion}

Fuzzy set theory is a powerful hand set for modelling uncertainty and vagueness in various problems arising in field of science and engineering. It has a wide range of applications in various fields. The concept of intuitionistic fuzzy normed space and of intuitionistic fuzzy 2-normed space is the latest developments in fuzzy topology. In the present paper we studied a more general type of paranorm intuitionistic fuzzy $I$-convergent sequence spaces defined by compact operator and study the fuzzy topology on the said spaces. These results provide new tools to deal with the I-convergence in intuitionistic fuzzy problems of sequences occurring in many branches of science and engineering.

\section{Acknowledgement}

The authors would like to record their gratitude to the reviewer for his careful reading and making some useful corrections which improved the presentation of the paper.

\section{References}

Alotaibi A, Hazarika B, and Mohiuddine SA (2014). On the ideal convergence of double sequences in locally solid Riesz spaces. Abstract and Applied Analysis, 2014: 1-6. http://dx.doi.org/10.1155/2014/396254.

Barros LC, Bassanezi RC, and Tonelli PA (2000). Fuzzy modelling in population dynamics. Ecological Modelling, 128(1): 27-33.

Das P, Kostyrko P, Wilczyński W, and Malik P (2008). I and I*convergence of double sequences. Mathematica Slovaca, 58(5): 605-620.

Edely OH (2003). Statistical convergence of double sequences. Journal of Mathematical Analysis and Applications, 288(1): 223-231.

Esi A and Özdemir MK (2016). On some real valued I-convergent $\Lambda$-summable diffrence sequence spaces defined by sequences of Orlicz functions. Information Sciences Letters, 5(2): 47-51. https://doi.org/10.18576/isl/050202

Fast H (1951). Sur la convergence statistique. In Colloquium Mathematicae, 2(3-4): 241-244

Fradkov AL and Evans RJ (2005). Control of chaos: methods and applications in engineering. Annual Reviews in Control, 29(1): 33-56.

Giles R (1980). A computer program for fuzzy reasoning. Fuzzy Sets and Systems, 4(3): 221-234.

Hazarika B and Mohiuddine SA (2013). Ideal convergence of random variables. Journal of Function Spaces and Applications, 2013: 1-7. http://dx.doi.org/10.1155/2013/ 148249.

Hong L and Sun JQ (2006). Bifurcations of fuzzy nonlinear dynamical systems. Communications in Nonlinear Science and Numerical Simulation, 11(1): 1-12.

Khan VA and Yasmeen K (2016a). Intuitionistic fuzzy zweier Iconvergent sequence spaces defined by Modulus function. Cogent Mathematics. Cogent mathematics (Taylors and Francis), 3(1): 1-10. 
Khan VA and Yasmeen K (2016b). Intuitionistic fuzzy zweier Iconvergent sequence spaces defined by Orlicz function. Annals of Fuzzy Mathematics and Informatics. Annals of Fuzzy Mathematics and Informatics, 12: 469-478.

Khan VA and Yasmeen K (2016c). Intuitionistic fuzzy zweier Iconvergent double sequence spaces. New Trends in Mathematical Sciences, 4(2): 240-247. https://doi.org/ 10.20852/ntmsci.2016218260

Khan VA, Ebadullah K, and Yasmeen K, and Fatima H (2014). On Zweier I-convergent sequence spaces, Proyecciones. Journal of Mathematics, 3(33): 259-276.

Khan VA, Esi A, Yasmeen K, and Fatima H (2017). On paranorm type intuitionistic fuzzy zweier I-convergent sequence spaces. Annals of Fuzzy Mathematics and Informatics, 13(1): 135-143.

Khan VA, Shafiq M, and Lafuerza-Guillen B (2015). On paranorm Iconvergent sequence spaces defined by a compact operator. Afrika Matematika, 26(7-8): 1387-1398.

Kostyrko P, Wilczyński W, and Šalát T (2000). I-convergence. Real Analysis Exchange, 26(2): 669-686.

Kreyszig E (1989). Introductory functional analysis with applications. Wiley, New York, USA.

Mohiuddine SA and Lohani QD (2009). On generalized statistical convergence in intuitionistic fuzzy normed space. Chaos, Solitons and Fractals, 42(3): 1731-1737.

Mursaleen M and Lohani QD (2009). Intuitionistic fuzzy 2-normed space and some related concepts. Chaos, Solitons and Fractals, 42(1): 224-234.
Mursaleen M and Mohiuddine SA (2009a). On lacunary statistical convergence with respect to the intuitionistic fuzzy normed space. Journal of Computational and Applied Mathematics, 233(2): 142-149.

Mursaleen M and Mohiuddine SA (2009b). Statistical convergence of double sequences in intuitionistic fuzzy normed spaces. Chaos, Solitons and Fractals, 41(5): 2414-2421.

Mursaleen M and Mohiuddine SA (2010). On ideal convergence of double sequences in probabilistic normed spaces. Mathematical Reports, 12(62): 359-371.

Mursaleen M, Mohiuddine SA, and Edely OH (2010). On the ideal convergence of double sequences in intuitionistic fuzzy normed spaces. Computers and Mathematics with Applications, 59(2): 603-611.

Mursaleen M, Srivastava HM, and Sharma SK (2016). Generalized statistically convergent sequences of fuzzy numbers. Journal of Intelligent and Fuzzy Systems, 30(3): 1511-1518.

Nabiev A, Pehlivan S, and Gürdal M (2007). On I-Cauchy sequences. Taiwanese Journal of Mathematics, 11(2): 569576.

Saadati R and Park JH (2006). On the intuitionistic fuzzy topological spaces. Chaos, Solitons and Fractals, 27(2): 331344.

Savas E (2004). On statistically convergent double sequences of fuzzy numbers. Information Sciences, 162(3): 183-192.

Zadeh LA (1965). Fuzzy sets. Information and control, 8(3): 338353. 